\title{
Gemeinwohlorientierung und Gemeinwohlrhetorik bei Parteieliten und Interessengruppen
}

\section{Das Netzwerk der Begriffe in der neueren Gemeinwohldiskussion der Eliten}

Der Titel, der die Gemeinwohlrhetorik ins Zentrum stellt, suggeriert, daß es ein Gemeinwohl nicht gibt, und daß es zu den „Gespenstern im Kornfeld" zur rechnen sei, welche der Ahnherr der Interessengruppentheorie, Arthur Fischer Bentley (1908) lächerlich machte. Er tat dies nicht ohne Heftigkeit, um seine Erfahrungen mit der Staatsmetaphysik als vormaliger Student an deutschen Hochschulen abzuschütteln und zum amerikanischen Pragmatismus zurückzukehren. Dennoch wird hier nicht unterstellt, daß das Gemeinwohl nur eine Leerformel ist. Es ist vielmehr eine normative regulative Idee, die nach Ausgleich der Interessen und nach Mindestanforderungen für eine gerechte Gesellschaft sucht. Gerechtigkeit ist daher im angelsächsischen und inzwischen auch im kontinentaleuropäischen Bereich seit der Rawls'schen Welle des Neokontraktualismus der präzisere Terminus. Gemeinwohl hatte lange einen Beigeschmack von katholischer Soziallehre und einem hierarchisch gedachten Altkorporatismus und blieb den calvinistischen Angelsachsen fremd. Blackwells Enzyklopädie der politischen Theorien ${ }^{1}$ verweist die Begriffe ,common good" oder ,public interest" in den Beitrag über „Interessen“. Die rational-choice-orientierte Einführung in eine „positive politische Theorie“ von Riker und Ordeshook ${ }^{2}$ kennt nur noch public goods, wie „Verteidigung und Leuchttürme", die im Sinne der Wohlfahrtsökonomie auf Pareto-Optimalität getestet wurden. Die Schwierigkeiten der Operationalisierung des Gemeinwohlbegriffs hat daher empirische Forscher bis hin zu Scharpf immer wieder auf mathematisierbare Nutzenkalkulation zurückgreifen lassen. ${ }^{3}$

Das öffentliche Interesse $e^{4}$ wurde in der Rechtswissenschaft als Begriff dem blassen Wort „Gemeinwohl" vorgezogen. Es zeigt sich als einer der meistgebrauchtesten For-

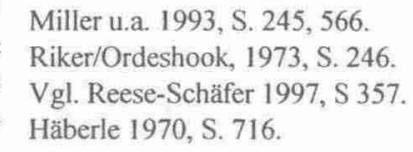


meln in der Rechtsprechung und Verwaltung. Gemeinwohl schien eine monarchischabsolutistische Formel darzustellen. Das öffentliche Interesse ist hingegen seine demokratisierte Form. Wo die Verfassung als öffentlicher Prozeß begriffen wurde, stellt sie zugleich eine normative Gemeinwohlordnung dar. Dies führt jedoch paradoxerweise zu einer Positivierung des Gemeinwohlbegriffs, der ihn als normativen Leitbegriff immer mehr aushöhlt. Kein Wunder, daß Häberle beklagen muß, daß es zwar eine Gemeinwohljudikatur in der Rechtsprechung gebe, daß diese aber niemals von der Wissenschaft systematisiert worden sei. Sie ist vermutlich durch inflationären Wortgebrauch nicht mehr zu systematisieren und verliert sich in der Kasuistik von Einzelfällen.

Hochkonjunktur hatte die Gemeinwohlformel bei der Gemeinde-Gebietsreform. Landesverfassungen und Gesetze ließen vielfach eine Gebietsänderung nur aus „Gründen des öffentlichen Wohls“ zu. Das öffentliche Wohl wurde dann mehr oder weniger ökonomisch und verwaltungstechnisch definiert. Auch Hans-Herbert von Arnim (1977), der immer wieder einer normativen Betrachtung der Interessentheorie das Wort redet, und als selbsternannter Anwalt des öffentlichen Interesses auch verdienstvoll entlarvend aufgetreten ist, hat das Gemeinwohl prozedural reduziert. Die Analogien zum Gerichtsprozeß sind in der Politik vielfach schief. ${ }^{5}$

Der demokratische Prozeß in seiner prozeduralen Fairness-Grundlage geht davon aus, daß anders als im Mittelalter major pars auch sanior pars ist. Es spricht bei jedem Mehrheitsbeschluß - ob knappe oder große Mehrheit oder gar Einstimmigkeit - eine Vermutung für Gemeinwohl. Mehrheit muß an die Stelle von Wahrheit treten.

Auf diese allgemeinen Grundsätze der Demokratie können sich heute alle Demokratien einigen. In der Genesis der europäischen Repräsentativsysteme wurden jedoch höchst unterschiedliche Konzeptionen darüber sichtbar, welche Akteure das Gemeinwohl formulieren durften.

In Deutschland hat es der Begriff des Interesses schwer gehabt, sich gegen die Metaphysik des Gemeinwohls und ein holistisches System der Staatsziele durchzusetzen. Es gelang erst mit der begrifflichen Trennung von Staat und Gesellschaft, etwa bei Lorenz von Stein. Noch bei Hegel waren Interessen als „Gegensatz" und „Kampf“ negativ bewertet. Nur der „allgemeine Stand“ konnte in seiner Rechtsphilosophie (§ 205) den allgemeinen Interessen dienen. „Die Verwaltung“ war selbst bei Forsthoff oder Werner Weber noch nach 1949 im „Maßnahmestaat" aufgerufen, das Gemeinwohl gegen die Habgier der Interessen zu verteidigen, da das Parlament von ihnen kolonialisiert schien. Autoritäre Vorstellungen, wie bei Carl Schmitt, daß die Exekutivspitze diese Aufgabe habe, ließen sich nach der Demokratisierung und Durchkonstitutionalisierung des Systems nicht mehr verfassungskonform vertreten. Ein Hauch des deutschen Etatismus fand sich noch bei einem Liberalen wie Theodor Eschenburg, ${ }^{6}$ der nach dem ,NSÜberstaat“ den „,demokratischen Unterstaat“ beklagte, der dazu führte, daß die Bundesrepublik nicht ein Bundesstaat, sondern ein „Gruppenbund“ sei.

In Frankreich hat das revolutionäre Pathos eines republikanischen Ideals den Gemeinwillen ins Zentrum gerückt. Die Rousseauisten glaubten, daß dieser Gerechtigkeit und Gemeinwohl erzeuge, sofern das Volk nicht irregeführt werde. In Großbritannien

\footnotetext{
Ähnlich schon: Schütt-Wetschky 1997, S. 2.
}

Eschenburg 1955, S. 81. 
wurde weniger „das Volk“ als „das Individuum“ als Hauptakteur zur Realisierung des Gemeinwohls angesehen - am krassesten im Utilitarismus.

Für Bentham ${ }^{7}$ war das Gemeinschaftsinteresse (,interest of the community ${ }^{*}$ ) in einer „fiktiven Einheit“ nur die Summe der Interessen der Mitglieder. Die utilitarische Lehre, die bis in moderne Rational-Choice-Ansätze herrscht, huldigt einem Wertrelativismus (Bentham drastisch: ,pushpin is as good as poetry“), der nur noch Präferenzordnungen rationaler Einzelakteure durchzurechnen erlaubt. In einer solchen Tradition kann das Bonmot eines genervten Amerikaners gedeihen, daß er seine Pistole entsichere, wenn er vom Gemeinwohl reden höre, weil er unterstellen müsse, daß man ihm mit einer solchen Ideologie nach Freiheit und Eigentum trachte. Bentham hatte immerhin ein „Handbook of rhetorical fallacies" verfaßt, das bis hin zu Ogdens „Meaning of the Meaning“, Stuart Chases „Tyranny of words“ oder Vance Packards „Geheime Verführer" zahlreiche Nachfolger hatte. Gemeinwohl gehörte in dieser Tradition, die im sprachtheoretischen Positivismus bei Weldon ${ }^{8}$ gipfelte, zu jenen Begriffen, die durch drei Illusionen verdächtig wurden: die Illusion realer Essenzen, die Illusion absoluter Standards und die Illusion einer geometrischen Methode zur Entdeckung solcher Wahrheiten.

Keines der drei Modelle zur Realisierung des Gemeinwohls galt exklusiv und zu allen Zeiten. Die Annäherung der angelsächsischen individualistischen Konzeption und der französischen demokratischen Gemeinwillen-Konzeption in den westlichen Demokratien hat auch in Deutschland dazu geführt, daß die Gemeinwohl- und Gerechtigkeitsanforderungen auf die Grundwerte reduziert worden sind.

\section{Wellen der Renaissance von Gemeinwohlrhetorik}

Nicht nur im transnationalen Vergleich gab es unterschiedliche Gemeinwohlkonzeptionen. Auch in einem Land wie der Bundesrepublik wurden je nach Akteur unterschiedliche Akzente und Begriffe gesetzt. In der Theorie dominierte der Gerechtigkeitsbegriff, bei den Parteien die Grundwerte, bei den Interessengruppen das Gemeinwohl und in der Gesetzgebung das „öffentliche Interesse“, vielfach desaggregiert zur Sozial-, Umweltoder Naturverträglichkeit.

In Deutschland lassen sich darüber hinaus vier Wellen eines normativen Räsonnements über das Gemeinwohl feststellen, die jeweils durch andere Grundbegriffe geprägt waren:

1. Eine Wiederbelebung der praktischen Philosophie und des Naturrechts nach 1945. In der stärksten Partei gab es einen Flügel, der die katholische Soziallehre wieder handlungsanleitend zu machen versuchte. Der liberale Mainstream, der eher sozialdemokratisch dachte, wie Ernst Fraenkel, ${ }^{9}$ vollzog die Synthese mit der angelsächsischen Tradition, die als Gegensatz zwischen Burke und Rousseau apostrophiert wurde. Gemeinwohl war aposteriorisch definiert, aber nicht nur Resultante im Interessenkampf. Gemeinwohl wurde

\footnotetext{
Vgl. Bentham 1984, Kap. I, 4.

8 Vgl. Weldon 1953 u. 1960, S. 20.

9 Vgl. Fraenkel 1964, S. 21, 42.
} 
zur regulativen Idee, nicht nur Reflex eines Überbaus des uniformierten und reglementierten Gemeinwillens.

2. Die Konzeption des Gemeinwohls als einer regulativen Idee, die nicht nur ein Konstrukt ex post facto darstellt, führte schon bei der Fraenkel-Schule zu einer Option für die Gemeinwohlsuche. Die Parteien fanden den kleinsten gemeinsamen Nenner in den Grundwerten. Die Suche nach neuen Werten in der Studenten- und Protestbewegung seit 1968 hat die Parteien und Verbände gezwungen, dem gewachsenen Bedürfnis der Gemeinschaft nach Normen wieder Rechnung zu tragen.

Seit dem ersten Konjunktureinbruch 1966 war die Gewißheit verflogen, daß das Gemeinwohl durch starke Umverteilungskraft des Staates naturwüchsig garantiert werden konnte. Erhards moralische Appelle an die Gemeinwohlorientierung der Interessen in einer „formierten Gesellschaft“ hatten sich als hohl erwiesen. Konkrete Organisation der Konsensfindung mußte in Angriff genommen werden und wurde in der Konzertierten Aktion und in neokorporativen Strategien gefunden. Im Stabilitätsgesetz (1967) wurden neue gemeinwohlfördernde Prozeduren gesetzlich festgeschrieben. Der Wirtschaftsminister glaubte das Wohlverhalten der Sozialpartner durch Mitwirkung „erkaufen“ zu können. ${ }^{8}$ Diese „Gemeinwohlverfahren" bewährten sich vorübergehend in Krisenzeiten. Sie waren aber ungeeignet für ,politics as usual“ - zumal die Konzertierung durch die Erweiterung der Teilnahmeberechtigung ihrer Schlagkraft beraubt wurde.

Der Grundkonsens der Aufbauzeit schien Mitte der sechziger Jahre zerfallen. Die anstehenden Probleme wurden 1966-69 durch einen organisierten Konsens der Volksparteien in der großen Koalition kompensiert. Herausforderungen durch den Terrorismus der siebziger Jahre und durch Berufsverbote, Hochschulreform, Änderungen des Demonstrationsrechts und Debatten um die innere Pressefreiheit nach Abhörskandalen zwangen zur normativen Neuorientierung. ${ }^{11}$

3. Der liberale Neokorporatismus hatte eine neue Form dezenter Staatssteuerung des Verbändewesens begünstigt. Kein Wunder, daß die Privilegierung der Tarifpartner und der großen Klassenorganisationen die Statusgruppen und die Liberalen ohne Zubringerorganisationen verschreckten. Der Neokorporatismus, der eine Weile Mode wurde, versandete in der schleichenden Krise einer Erosion der Steuerungsfähigkeit des Staates. Der Regierungswechsel von 1982 hatte die konservativen Statusängste über Nacht zerstreut. Neue Probleme standen an.

4. Die neoliberale Welle, die Entstaatlichung, Deregulierung und Privatisierung förderte - wenn sie auch auf dem Kontinent keine Konsequenz à la Thatcher und Reagan hatte schuf neue soziale Ungleichgewichte. Die Rhetorik des demokratischen Sozialismus mit seinen etatistischen Regulierungsrelikten, denen von gegnerischer Seite ein Bismarckbias für Sozialpolitik als rein staatliche Veranstaltung unterstellt wurde, kam in vielen Ländern in die Defensive. Eine Welle der Verteidigungsrhetorik gegen den Neoliberalismus dominierte, bis in den neunziger Jahren die totgesagten sozialdemokratischen Parteien in vielen Ländern wieder an die Macht kamen.

${ }^{10}$ So von Arnim 1977, S. 143.

11 Vgl. Schwan 1978, S. 339. 
Eine neue Gemeinwohldebatte setzte ein. Die Sozialdemokraten bekamen unerwartete Schützenhilfe aus den USA. Der Kommunitarismus hatte dort Front gegen einen unkontrolliert ins Kraut schießenden Liberalismus gemacht. Rawls als Liberaler hatte 1971 eine neue Gerechtigkeitsdebatte entfesselt, an der Europa überwiegend nur rezeptiv teilnahm.

Der Nachkriegskonsens hinsichtlich der Gemeinwohldebatte hatte sich prozedural verengt auf Öffentlichkeit, Partizipation aller Bürger und dem Zwang, in einer deliberativen Demokratie seine Gründe darzulegen. Nun wurde mit, ,justice as fairness" zunächst nur ein halbherziger Schritt über das prozedurale Verständnis von Gerechtigkeit hinaus gewagt. Aber der neue Egalitarismus der Gerechtigkeit ging weiter als die Chancengleichheit des herkömmlichen Liberalismus und zielte auf Gleichheit in den Ergebnissen ab. Der Kommunitarismus hat gegen die alten a posteriori-Definitionen des Pluralismus a priori Grundsätze der guten und gerechten Ordnung wieder ins Gespräch gebracht. Erhalten blieb dabei die Kritik am Vorrang der individuellen Rechte. Die Integration über das Recht reichte den Kommunitaristen nicht aus. Sie bestanden auf Gemeinschaftswerten, ohne aber den Rückgriff auf das familiale Ethos eines Tugendstaates oder einer ethnisch verstandenen nationalen Gemeinschaft anzustreben.

Wie in den siebziger Jahren der Kritische Rationalismus als Gegengewicht gegen den ideologischen Marxismus zur Staatsphilosophie aller Parteien wurde, zuerst der Liberalen dann auch der beiden Volksparteien, so wurde der Kommunitarismus in den achtziger Jahren von den Parteien als Schützenhilfe benutzt, um die stagnierende Gemeinwohl- und Grundwertedebatte wieder zu beleben. Kommunitarier wurden auf Parteitagen und Programmkommissionen herumgereicht. Je stärker sich Kommunitaristen auf konkrete Programme einließen und praktische Modelle förderten, wie Etzioni, ${ }^{12}$ kamen sie zurück zu einer wenig amerikanischen Kritik an der Lobbypolitik, welche die Politik ,gekauft" und „verdorben" habe und gerierten sich als public interest group, die für das Gemeinwohl stritten, ohne etwas für sich selbst zu fordern.

Die Gegenreaktion blieb nicht aus. Der Kommunitarimus wurde als sympathischer „Antiliberalismus auf Samtpfoten“ verdächtigt, ${ }^{13}$ der mit der Gemeinschaftsverherrlichung à la Hannah Arendt in, gleichsam unbeflecktem Gewand zurückkehrt und von deutschen Intellektuellen scheinbar reinen Gewissens wieder aufgegriffen werden kann." Die alte Gegenüberstellung von Gemeinschaft und Gesellschaft seit Tönnies schien mit dieser Bewegung wieder aufzuleben. Theorien der Zivilgesellschaft konnten den neuen Idealismus verbrämen. Allenfalls das Korrelat auf der Operationalisierungsebene, die citizenship mit der Differenzierung als rechtliche, politische, soziale und kulturelle Bürger, konnte den Druck zu einer neuen Gemeinschaftstyrannei wieder mildern.

12 Vgl. Etzioni 1995, S. 245 ff.

13 Herzinger 1997, S. 115. 


\section{Die Differenzierung der Gemeinwohlrhetorik der politischen Eliten}

Zwei Ebenen der Interessenvertetung wurden zur Arena von Gemeinwohldebatten: die Parteien und die Interessengruppen. In einer konsolidierten Demokratie gilt es als Postulat, daß beide sich ausdifferenziert haben sollten. Eine allzu enge Filzokratie zwischen Parteien und Verbänden gilt in der politischen Rhetorik als gemeinwohlgefährdend. In Deutschland richtete sie sich vor allem gegen die enge Verbindung von Gewerkschaften und SPD, obwohl es niemals kollektive Mitgliedschaften in der SPD gab, wie in der Labourparty und früher in einzelnen sozialdemokratischen Parteien Skandinaviens. Auch im bürgerlichen Lager, in der Österreichischen Volkspartei bis heute und anfangs auch in der CSU, hat es solche kollektiven Verflechtungen gegeben.

Parteien sind schon vom Namen her ,pars " und daher nicht das Ganze. Das hat einige nicht gehindert, als „Union", „Bewegung", „Rassemblement" und in anderen Bezeichnungen, volksumspannende Ansprüche zu signalisieren. Die Parteien sind von Schweden bis Italien und Spanien heute partiell staatlich alimentiert und als Akteure der Teilnahme an der staatlichen Willensbildung wenigstens auf eine prozedurale Gemeinwohlkonzeption verpflichtet. Konsequenterweise konnte es ein Parteiengesetz, nicht aber ein Verbändegesetz geben.

Mit zunehmender Entzauberung des Staates und dem wachsenden Gewicht der Implementation über die Gesetzgebung sind ältere Vermutungen, daß Verbände ebenfalls in öffentliche Funktionen hineinwuchsen, nicht von der Hand $\mathrm{zu}$ weisen ${ }^{14}$ - nicht erst seit das private interest-government entdeckt wurde.

Dennoch haben Politikwissenschaftler ${ }^{15}$ die Parteien als die wichtigsten Antriebskräfte der konkreten Gemeinwohlverwirklichung angesehen. Soweit sie Politik formulieren und gestalten, sind sie in der Tat am stärksten an jener Resultante im Kräfteparallelogramm beteiligt, die im Nachhinein bei der Entscheidung als das Gemeinwohl angesehen wird. Da es meist eine Opposition gibt, die in vielfacher Hinsicht auf die Entscheidung einwirkt ${ }^{16}$ ist eine Entscheidung im parlamentarischen Parteienstaat vergleichsweise gemeinwohlnäher als wenn Interessengruppen hinter verschlossenen Türen einen sozialverträglichen Kompromiß ausgehandelt hätten.

Die Parteien - selbst nur ,partes" der Gesellschaft - nehmen in ihrer Programmatik vielfach eine gönnerhafte Haltung gegenüber den Verbänden ein, als ob sie die Gemeinwohlorientierung von Organisationen am besten beurteilen könnten. Während das CDU-Grundsatzprogramm von 1978 nur die ,Sozialpflichtigkeit aller gesellschaftlichen Kräfte" beschwor (Tz. 122), verstieg die SPD sich in ihrem Grundsatzprogramm von 1989 (S. 48) zu dem Satz „Verbände sind legitimer Ausdruck gesellschaftlicher Interessen. Wo sie sich am Gemeinwohl orientieren, suchen wir mit ihnen Zusammenarbeit. Wo sie rücksichtslos Teilinteressen durchsetzen wollen, treten wir ihnen entgegen". Sowie die SPD an der Macht war, mußte sie gerade mit jenen orga-

\footnotetext{
14 Teubner 1978, S. 118.

is Vgl. Schwan 1986, S. 861.

16 Beyme 1997, S. 92 ff., 263 ff.
} 
nisierten Interessen kooperieren und nicht nur ihnen entgegentreten, unter denen sie auf wenig Konsens für ihre Gemeinwohlvorstellungen hoffen konnte. Man denke an Ärzte und Pharmaindustrie, aber zum Teil auch an die Krankenkassen in den Konflikten um die Gesundheitsreform.

In den USA wurden schon lange die iron triangles der Mitentscheider von Parlamentariern, Bürokraten und Interessengruppen als normal angesehen. In Europa weitete sich das Netzwerk zu ungemütlichen Vier- oder Fünfecken aus: es traten die Parteien in größerem Maß als in den USA formend hinzu und in Deutschland und anderen föderalen Staaten die Länder. ${ }^{17}$ Die Netzwerkansätze der politischen Entscheidung werden vermutlich langfristig auch bei uns ein hierarchisches Modell der Mitentscheidungsbefugnis qua angeblicher Gemeinwohlnähe obsolet werden lassen. Die Not wird in der Wissenschaft längst zur Tugend umdefiniert, weil horizontal vernetzte Verbändesysteme im postmodernen Staat als bloßem „Mediator" dem Ideal der ,gesellschaftlichen Selbstregulierung" am nächsten kommen. ${ }^{18}$

Die Wissenschaft hat auf der Suche nach dem öffentlichen Interesse zum Teil an der patronisierenden Haltung der Parteien gegenüber den Interessengruppen mitgewirkt. Glendon Schubert ${ }^{19}$ unterschied die Idealisten, die ,pro-public " und gegen Parteien und Interessengruppen eingestellt waren, und die Realisten, die „pro-interest group“ optierten, ohne Anti-Parteien-Affekte zu zeigen. Sie sahen in ihnen jedoch auch nur eine Art von „Interessengruppen“ auf anderer Ebene.

Die patronisierende Haltung der Parteien gegenüber den Verbänden ist im Vergleich zu den siebziger Jahren hinsichtlich der Gemeinwohlrhetorik geringer geworden. Neben der positiven Hinwendung zu Grundwerten und Gemeinwohl-Appellen standen in den siebziger Jahren die negativ-defensiven Varianten der Gemeinwohldebatte. Ausgerechnet die Liberalen begannen eine Diskussion um das Verbändegesetz, die wenig zu den Prämissen ihrer Parteiideologie zu passen schien. Diese erklärt sich vermutlich aus der Zeit der großen Koalition, als die FDP als Miniopposition einen einsamen Kampf gegen die Giganten führte. Diese hätten um ein Haar das Wahlrecht geändert, und das hätte die Partei vermutlich das Leben gekostet. Zudem wuchsen die beiden Volksparteien in der Zeit hektischer Mobilisierung nach der Studentenrevolte und profitierten von ihren Zubringeorganisationen und organisierten Klientelgruppen. Die enge Kooperation von Parteien und Verbänden geriet ins Visier der Kritik, und es wurde versucht, die Gemeinwohlpflichtigkeit der Verbände gesetzgeberisch zu fördern. Ein Verbändegesetz wurde von einer Kommission des Bundesvorstands der FDP 1976 in Murrhardt vorgelegt. Die CDU schien in ihrer Mannheimer Erklärung von 1975 dem Gedanken nicht abgeneigt zu sein, hat sich aber im Grundsatzprogramm 1978 zu der Einsicht durchgerungen: ,Ein Verbändegesetz lehnen wir ab. “20 Ansatzpunkt war vielfach die Vorstellung einer weiteren Demokratisierung der innerverbandlichen Strukturen. Wie beim Parteiengesetz drohte jedoch ein solches Gesetz in einen Teil allgemeiner Selbstver-

\footnotetext{
17 Vgl. Beyme 1997.

18 Mayntz 1992, S. 34.

19 Schubert 1960, S. 200 f.

${ }^{20}$ Texte in: von Alemann/Heinze 1979, S. 214; Darstellung in Göhner 1981, S. 16 ff.
} 
ständlichkeiten und in einen besonderen Teil, der ein reines Reglement der Finanzierung der Organisationen darstellte, zu zerfallen.

In der SPD und den Gewerkschaften wurde in diesen Plänen eine antigewerkschaftliche Stoßrichtung vermutet, die sich am Popanz eines angeblichen Gewerkschaftsstaats entzündete. Selbst eine unterhalb eines Gesetzes angesiedelte Verrechtlichung der Arbeitsbeziehungen galt schon als „Verbändegesetz auf Raten“. ${ }^{21}$ Das ubiquitäre organisatorische Gemeinwohlrelikt aus der Zeit des älteren Korporatismus, die Forderung nach einem Sozial- und Wirtschaftsrat, wie er in der Weimarer Republik existiert hatte und in vielen Demokratien von Holland bis Italien bestand, wurde nun eher suspekt. Der DGB betonte diese Forderung seines Programms nicht mehr. Der Union wurde die Ablehnung eines Verbändegesetzes erleichtert durch die Stellungnahme der Unternehmerverbände gegen eine „ständestaatliche Tendenz“. Rolf Rodenstock formulierte 1976 bei den Pyrmonter Unternehmergesprächen, ${ }^{22}$ daß diese die Symmetrie der Tarifpartner und ihrer Autonomie stören müsse. Die Gewerkschaften wurden aber gewarnt, sollten sie ihre Zuständigkeitsvermutung über die Arbeitsmarkt-, Tarif- und Sozialpolitik hinaus auszudehnen und sich über eigene Firmen und mit politischer Pressure immer mehr in die allgemeine Wirtschaftspolitik einzumischen. Mit dem Heraufkommen des Korporatismus versandete die Debatte rasch im ausgetrockneten Flußbett der politischen Nichtentscheidungen.

Der Aufstieg des Neoliberalismus veränderte das „Werteklima“. Mandevilles Bienenfabel, ${ }^{23}$ nach der auch die Faulsten und Bösartigsten gezwungen sind, für das Gemeinwohl tätig zu werden, kann im Neoliberalismus nicht mehr so unverblümt geglaubt werden. Die Gerechtigkeitsvorstellungen ökonomisierten sich zwar; ${ }^{24}$ aber je härter die Verteilungskämpfe wurden, umso ausgefeilter wurde die Gerechtigkeits- und Gemeinwohlrhetorik der Parteien und Interessenten.

Eine positive Hinwendung zur Gemeinwohlrhetorik vollzog sich in der Operationalisierung von Gemeinwohlvorstellungen in einigen Grundwerten. In der staatsrechtlichen Diskussion hatte es immer schon mehrere Gemeinwohlgrundwerte gegeben, wie Freiheit, Gerechtigkeit, Sicherheit, Frieden und Wohlstand. ${ }^{25}$ Ihre Zahl mußte in der Selbstdarstellungsrhetorik zur besseren Wirksamkeit für Parteien reduziert werden. Grundwerte waren nach der aufgeklärten Auffassung der Parteien nicht mehr vom Staat erzeugt, sondern dieser war der „Empfänger der letzten Werte und Grundwerte" (Helmut Schmidt). ${ }^{26}$

Die Gerechtigkeit wurde zum Zentralbegriff der Theoriebemühungen aller Parteien. Aber es herrschten durchaus unterschiedliche Auffassungen von Gerechtigkeit. Ein jüdisch-spanischer Philosoph, Shem-Tov Ibn Falaquera (1225-1295), ${ }^{27}$ hat drei Gerechtigkeitskonzeptionen in eine Fabel gepreßt: Zwei Wanderer lassen sich zum Imbiß nie-

DGB-Antrag 1978; von Alemann/Heinze 1979, S. 257.

22 Ebd., S. $258 \mathrm{ff}$.

23 Mandeville 1968, S. 134.

${ }^{24}$ Münkler/Llanque 1999, S. 9.

${ }^{25}$ Arnim 1977, S. 22 ff.

${ }^{26}$ Zit. nach Sarcinelli 1979, S. 51.

27 Vgl. Falaquera 1976. 
der. Einer hat drei, der andere zwei Brote. Ein Dritter tritt hinzu und begehrt, mit zu essen - gegen gute Bezahlung. Am Ende verabschiedet er sich und gibt fün Dinar mit der Maßgabe, die beiden sollten sie ,gerecht" teilen. Der Inhaber von drei Broten verlangte die gerechte Aufteilung drei zu zwei. Das Modell wurde aristotelisch genannt: jedem das Seine. Derjenige, der nur zwei Brote eingebracht hatte, erklärte sich für eine egalitäre Gerechtigkeit. Jeder sollte zweieinhalb Dinar bekommen. Da sie sich nicht einigen konnten, gingen sie zu einem Richter. Der entschied die Aufteilung 4:1 zugunsten des Inhabers von drei Broten. Die marktwirtschaftliche Gerechtigkeit war entdeckt. Jeder der drei aß ca. 1.7 Brote. Der Inhaber von zwei Broten hat ca. 0.3 Brote über den Eigenverzehr hinaus auf den Markt gebracht, etwa ein Viertel des anderen, der 1.3 Brote für den Markt freigab. Vier zu eins schien somit „,marktgerecht“.

Die drei Konzeptionen konkurrieren auch im Streit der Parteien. Die egalitärkommunistische Auffassung hat an Boden verloren. Aber die aristotelische Konzeption konkurriert noch immer mit der neoliberal-marktwirtschaftlichen Konzeption. Dennoch haben die Gerechtigkeitsvorstellungen sich angenähert. Keine der beiden Volksparteien vertritt noch eine reine Modellvorstellung von Gerechtigkeit, sobald die wohlfahrtsstaatlichen Korrektive der Gerechtigkeit debattiert werden.

Zum Teil wurde die Debatte in den Parteien von Gruppen, die hinter ihnen standen, ausgelöst, wie bei der Union vom Zentralrat der Katholiken. Im Ringen um die Prinzipien eines demokratischen Sozialismus und der Aufgabe der marxistischer Ideologie kam es bei der SPD zu einer Grundwertedebatte. Das Godesberger Programm (1959) hat als Konkretisierungen den Dreiklang Freiheit, Gerechtigkeit und Solidarität kanonisiert. Solidarität war dabei der am stärksten aus der Arbeiterbewegung beglaubigte Begriff.

Im Orientierungsrahmen 1975-85 wurden die christlichen und humanistischen Wurzeln in gleicher Weise betont und Solidarität in Freiheit ohne Zwänge herausgestellt. Im Grundsatzprogramm der CDU von 1978 wurden schließlich die gleichen drei Werte verankert. Dies verstärkte die Kritik an der ideologischen Gesichtslosigkeit der Volksparteien. $^{28}$

Die Sozialdemokraten betonten stärker als die Christdemokraten die Gleichrangigkeit aller drei Prinzipien. Aber beide großen Volksparteien hatten über die Kompatibilität der drei Prinzipien und ihre möglichen Widersprüche nicht hinreichend nachgedacht. Solidarität ist ein Prinzip, das man allenfalls erbitten kann. Gerechtigkeit kann man verlangen. Über mangelnde Solidarität gibt es Enttäuschungen. Mangelnde Gerechtigkeit löst Empörungen aus ${ }^{29}$ und kann zu schweren Störungen der öffentlichen Ordnung führen. Solidarität greift vor allem bei unverschuldeter Benachteiligung von Gruppen. Gerechtigkeit als Prinzip gilt auch bei mitverschuldeter Not. Insofern zeigt sich die Solidarität als stärker gruppengebunden und auf Minderheiten bezogen. Die Sozialdemokratie bezog sich auf die Solidarität der Arbeiterklasse, die sie für tendenzielle Mehrheit erklärte, ohne daß sie das als subjektive „Klasse für sich“ je geworden ist. Die Christdemokratie bezog sie anfangs auf die Mehrheit der Christen, die jedoch von einer tendenziellen Gesamtheit in der säkularisierten Welt immer stärker zu einer Minderheit

\footnotetext{
28 Vgl. Meyer 1981, S. 9.

$29 \mathrm{Vgl}$. Höffe 1995.
} 
wurde. Die alte jakobinische Trinität Freiheit, Gleichheit, Brüderlichkeit wurde bei den Grundwerten verändert. Gleichheit schien weniger wichtig als Freiheit. Auch Rawls, ${ }^{30}$ der das Staatsschiff mit einem Schiff auf stürmischer See verglich, hat eine Gemeinwohlvermutung für Kapitän und Regierung sprechen lassen: die Gleichheit wurde vermindert, um das Gemeinwohl zu mehren!

Die Freiheit wurde in einer konsolidierten Demokratie vorausgesetzt und es gab nur noch kleine Minderheiten, die sie in Frage zu stellen wagten. Die Gerechtigkeit aber wurde zum Zentralbegriff und war von den Parteien umstritten. Die Rangfolge der drei Prinzipien ist für die empirische Politikforschung nicht belanglos. Gerechtigkeit ist zweifellos das Prinzip, das am stärksten die Legitimationsgefühle der Bürger bestimmt. Eine Verletzung der elementaren Gerechtigkeitskriterien schwächt die Zustimmung zum politischen System stärker als einzelne Einschränkungen der Freiheit, wie das Wachstum des Überwachungsstaats zeigt.

Es ist vielleicht kein Zufall, daß die Zustimmung zu den Institutionen, die über Recht und Gerechtigkeit wachen - Gerichte und Polizei - nach den Umfragen in ganz Europa am größten ist. Keineswegs nur in Deutschland, dem seit der Civic Culture-Studie ${ }^{31}$ ein legalistisches Bias nachgesagt wird, das auf einen Angstkomplex vor der Politik zurückgeführt worden ist.

Die Übereinstimmung hinsichtlich der drei Grundwerte in den beiden großen Volksparteien ist gewachsen. Differenzen bleiben hinsichtlich der Vorstellung, wie weit diese Werte mit staatlicher Hilfe zu realisieren seien. Der Vorsitzende der SPD-GrundwerteKommission Eppler ${ }^{32}$ sah eine Gefahr darin, daß die Grundwerte, die allen Parteien gemeinsam sind, zu abstrakt bleiben und, über dem politischen Tagesgeschäft thronend, nicht handlungsanleitend werden.

Die Subsidiarität wurde in der Christdemokratie stärker betont, um dem etatistischen Zentralismus vorzubeugen. Als Kompromißformel eignete sich die Subsidiarität bei der Vorbereitung der Maastricht-Akte vorzüglich, den widerstrebenden Briten, die Sorge vor der Vokabel Föderalismus hatten, einen wenig präzisen und im britischen Protestantismus nicht geläufigen Formelkompromiß anzudienen. Der instrumentale Charakter der Formel trat zutage, als die Mehrheit des Parlaments in Straßburg präzisierte, daß Subsidiarität im Zweifel Föderalismus bedeute.

Bei der SPD kam trotz Godesberg in Sonntagsreden ein eschatologisches Moment auf, nach dem sich „Demokratie nur im Sozialismus erfüllt" . ${ }^{33}$ Gegen eine Vorstellung, daß Gemeinwohl und Grundwerte eigentlich erst auf einer höheren sozialistischen Stufe der Evolution zu erreichen seien, hatte die Union ein leichtes Spiel der Zurückweisung. Obwohl beide Volksparteien einst den Fallibilismus des Kritischen Rationalismus akzeptiert hatten, wurde er nun gegen die SPD ausgespielt: „Jede Gruppe und jede Partei kann irren. Im demokratischen Wettbewerb der Ideen und Programme findet das freie

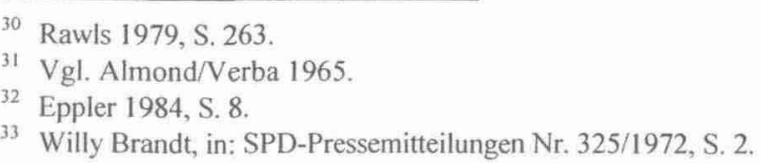


Gemeinwesen am besten zu sich selbst. Die Grundwerte aber, auf denen es beruht, bewähren sich nur in der Verbindlichkeit für alle." ${ }^{34}$

Mit zunehmender Erstarkung des Neoliberalismus in den neunziger Jahren geriet die SPD in Verdacht, in eine Gerechtigkeitsfalle geraten zu sein. Die SPD wollte nicht nur vor dem „Wind der Evolution" hertreiben, sondern das Schiff der Gesellschaft notfalls gegen den Wind kreuzen lassen. ${ }^{35}$ Was bei ihren Anhängern als Tugend galt, schien den Gegnern der SPD als Beleg dafür, daß die SPD weiterhin eschatologisch nach Deutungshoheit für die Gerechtigkeit strebte. Besitzstandswahrung und Interessenpolitik wurden als Gerechtigkeit ausgegeben. Sozialverträglichkeit wurde als Verhüllung des Partikularinteresses angesehen, von dem Schröder so häufig sprach. ${ }^{36}$

Je größer die potentielle Gruppe, für die ein Verband spricht, umso stärker die Neigung, das eigene Interesse mit dem Gemeinwohl zu identifizieren. Der Bund der Steuerzahler ist ein gutes Beispiel. Jeder möchte wenig Steuern zahlen. Prima vista vertritt der Bund das Interesse von jedermann. Denkt jedermann aber nach und versucht, seine sonstigen Ziele gegen den Steuerersparniswunsch aufzurechnen - gute öffentliche Bildungsanstalten für seine Kinder, gute ärztliche Versorgung für seine Familie etc. -, so könnte das Steuersparen um jeden Preis doch nicht jedermanns Ziel sein.

Zudem kann man sich mit einigen Verbandszielen leichter identifizieren als mit anderen. Die Aufrechnungen, welche Verschwendungen es in der Verwaltung pro Jahr gegeben hat, sind sicher konsensfähiger als bestimmte Steuerersparnisvorschläge, die sogleich an den Grenzen der Parteiideologien rütteln, mit denen jedermann sich unter Umständen identifiziert. Jeder Verband mutet seinen Mitgliedern im Namen des Gemeinwohls gelegentlich Konzessionen zu. Er tut dies jedoch nur, wenn es dem Kern der Mitglieder finanzielle Vorteile zu bringen verspricht. ${ }^{37}$

Es ist nicht Aufgabe von Verbänden, gemeinwohlorientiert zu sein. Vermutlich ist dies mit ihren Aufgaben nicht vereinbar. ${ }^{38}$ Jedes egoistische Interesse - wie auch das der Parteien - hat jedoch ein Interesse an der Erhaltung des Systems einer friedlichen Konfliktaustragung und am System einer funktionierenden Marktwirtschaft. Diese verallgemeinerbaren Systemerhaltungsinteressen Gemeinwohl zu nennen ist möglich, aber nicht sehr ergiebig. Schwieriger ist die Entlarvung als interessengebunden und nicht gemeinwohlpflichtig bei public interest groups, die schon im Namen ihre Nähe zum public good suggerieren.

In der Typologie der Interessengruppen wurden schon früh die promotional groups oder ideellen Fördererverbände von den Gruppen mit wirtschaftlichen und finanziellen Interessen gesondert. In Amerika waren jedoch selbst die Glaubensgemeinschaften nicht strikt als meta-wirtschaftlich zu deklarieren. Bei Scientology wurde sogar von einer religiösen Verbrämung eines rein wirtschaftlichen und machtpolitischen Interesses ausgegangen. Durch die neuen sozialen Bewegungen entstand ein neuer Typ von public interest group, der durch Advokatenpolitik zugunsten unterprivilegierter Gruppen der

${ }^{34}$ Geißler 1979, S. 179.

35 Dohnanyi 1999, S. 41.

${ }^{36}$ Ulrich 1999, S. 31

${ }^{37} \mathrm{Vgl}$. Heinze 1992, S. 123.

${ }^{38} \mathrm{Vgl}$. Groser 1992, S. 26. 
Gesellschaft seine Uneigennützigkeit und Gemeinwohlnähe besser glaubhaft machen konnte als viele der älteren promotional groups. Im Modell eines vollständigen Pluralismus mit geringen Zulassungsbarrieren sind diese relativ stark - im Vergleich zu den meisten kontinentaleuropäischen Ländern mit einer Dominanz der großen Klassen- und Statusorganisationen.

Olson falsifizierte die Annahme der alten Bentley-Truman-Doktrin, daß jedes Interesse auch zu einer Organisation finde. Die normalen Interessengruppen, die vor allem für das Wohl einer begrenzten Klientel eintreten, die seit Olson ${ }^{39}$ als besonders erfolgreich galten, je begrenzter ihre Organisationsziele sind, bedürfen zu ihrer Durchsetzung der Inszenierung und der Suche nach Verbündeten. Die Gemeinwohlnähe wird in den Programmen vielfach suggeriert: Bauernverbände pflegen die Landschaft und produzieren "gesunde Nahrung“, obwohl inzwischen die Landwirtschaft als einer der ökologischen Hauptsünder entlarvt wurde. Der Appell an die Gemeinwohlorientierung findet sich auch bei sehr partiellen Wirtschaftsverbänden: ${ }^{40}$ Sie verführt zur Ausweitung der Ziele, etwa wenn Gewerkschaften sich als Sprecher von arbeitenden Produzenten und der Mehrzahl der Konsumenten deklarieren, obwohl das Konsumenteninteresse durchaus eine Tarif- und Sanktionspolitik gegen einzelne Betriebe verlangen kann, deren Politik von den Gewerkschaften gedeckt wurde. Der Gegenverband brandmarkt solche Ausweitungen gern als Gefahr für die Zukunft. „Unternehmer- oder Gewerkschaftsstaat" werden dann als Anklagepunkte ausgetauscht, die das Gemeinwohl verletzen.

Der rein instrumentelle Gebrauch der Gemeinwohlrhetorik zu vordergründigen Machtspielen ist freilich weniger interessant geworden. Eine neue Dimension erhalten die Gemeinwohlappelle in ihrer reduzierten Form in der Risikogesellschaft und in einer Gesellschaft sozialer Verwerfungen durch Europäisierung und Globalisierung. Historische Gemeinwohlbeschwörungen in Huldigungs- und Vereidigungsformeln sprachen schon immer davon, daß der Herrscher das „Wohl des Landes zu mehren“ und „Schaden von ihm abzuwenden" habe. In der Risikogesellschaft wurde der zweite Aspekt fast wichtiger als der erste.

In der dritten Welle der Gemeinwohlrhetorik hat sich die Thematisierung der Risiken in die Organisation selbst hinein verlegt.

Noch immer wird die Deckungsgleichheit des eigenen Interesses mit dem Gemeinwohl oder die Identität des eigenen Interesses mit dem der großen Mehrheit oder wenigstens die Funktionalität des eigenen Interesses für eine „nachhaltige Entwicklung“ der Gesellschaft behauptet. ${ }^{41}$ Vielfach gilt die EU-Verträglichkeit a priori als gemeinwohlträchtig und die nationale oder regionale Interessenverfolgung als unzeitgemäß.

Die Gemeinwohlphraseologie partialisiert sich und ist zugleich mit dem zukunftsschwangeren Unterton vermeidbarer Risiken verknüpft. Am häufigsten sind die Reduzierung der Gemeinwohlanmaßung auf die Umweltverträglichkeit und auf die Sozialvertäglichkeit von eigenen Forderungen. ${ }^{42}$ Die Gefahr dabei ist, daß kleine aufgeklärte

\footnotetext{
${ }^{39} \mathrm{Vgl}$. Olson 1968.

${ }^{40}$ Hondrich 1963, S. 134.

41 Vobruba 1992, S. 105.

${ }^{42}$ Ronge 1992, S. 50 ff.
} 
Gruppen nach Mißerfolgen immer häufiger im Sinne von Hirschman ${ }^{43}$ nicht voice sondern exit, nicht Engagement sondern enttäuschten Rückzug wählen.

\section{Die empirische Kontrolle über die Gemeinwohlträchtigkeit von Entscheidungen}

Der Empiriker bleibt selbst von empirischen Studien über Gemeinwohlrhetorik unbefriedigt. Das Festhalten an einem Gemeinwohlpopanz zu strategischen Zwecken ist kein origineller Befund, so wenig wie die Feststellung von Kriegsgeschrei in alten Indianerkämpfen. Wie kann der Einsatz überprüft werden? In der politischen Debatte wie in der wissenschaftlichen Literatur werden drei Indikatoren für die Gemeinwohlvermutung eingesetzt, die um die Begriffe der „Repräsentativität und Inklusion“, der „qualifizierten Mehrheit" und des „wissenschaftlich unterstützten Sachverstandes“ kreisen: a) die Inklusivität des Systems der Interessenrepräsentation, b) die Mehrheiten, die eine Maßnahme tragen, und c) die wissenschaftliche Vorbereitung zur Vermeidung von Schädigungen des Gemeinwohls und die Ergebnisse der ex-post-Evaluation

\subsection{Die Inklusivität des Systems der Interessenrepräsentation}

Die Inklusivität eines Systems der politischen Entscheidung gilt als Wertmaßstab. Interessenvermittlungssysteme sind umso gerechter, je inklusiver sie sind und je mehr unterschiedliche Interessen Berücksichtigung finden. ${ }^{44}$ Die üblichen Typologien von korporatistischen und pluralistischen Systemen verdecken eine komplexe Realität. Je nach Maßnahmetyp und Politikfeld sind die Mischungsverhältnisse der organisierten Interessen, die sich zu artikulieren versuchen, unterschiedlich. Es fehlen vergleichende Studien. Für Deutschland - in internationalen Klassifikationen als gemäßigt korporativ eingestuft - zeigte sich bei 150 Gesetzen in 12 Bundestagen ein differenziertes Bild.

Nur bei $12.7 \%$ der untersuchten Gesetze, vornehmlich in der Rechts- und in der Baupolitik, ist eine Vielfalt von Statusgruppen in der Entscheidungsarena des Bundestages präsent. In weiteren $15.5 \%$ der Gesetzesvorhaben ließ sich eine Dominanz der Förderergruppen und public interest groups feststellen. Diese Fälle waren aber zu $87 \%$ auf die Rechts- oder die Umweltpolitik konzentriert. In einem Viertel der Fälle ist der Zugang auf die korporativen Großorganisationen beschränkt. In über der Hälfte der Entscheidungen liegt eine Privilegierung der korporativen Großgruppen und der großen Statusgruppen vor, die eine Art Interessenoligopol bilden. Die Inklusivität der Interessenartikulation ist in Deutschland geringer als in den USA, aber größer als in Frankreich, wo staatliche Akteure eine wichtige Funktion im System der ,pantouflage " haben, oder in Italien mit seinen Parentela- und Clientela-Verhältnissen zwischen staatlichen und

$43 \mathrm{Vgl}$. Hirschman 1984.

44 Vgl. Abromeit 1993, S. 10. 
gesellschaftlichen Akteuren. Je stärker etatistisch die Interessenarenen zugeschnitten sind, umso geringer die Inklusion. ${ }^{45}$

Die Zahl der Eingaben und Teilnahmen an Hearings gestattet noch keinen Zusammenhang zwischen Interventionsversuch und Erfolg der Durchsetzung in einem Gesetzentwurf zu konstruieren. Dieser ist allenfalls durch Textvergleiche von Eingabe und Gesetzestexten möglich. Die Gemeinwohlrhetorik der Rechtsprechung ist vergleichsweise leichter zu bearbeiten. Die Einflüsse der Gemeinwohlrhetorik sind hier nur auf ihre ideologischen Grundlagen zu untersuchen. Es muß kein direkter Einfluß organisierter Interessen unterstellt werden.

\subsection{Die Mehrheiten, welche Entscheidungen tragen}

Das Prinzip „Mehrheit statt Wahrheit“ liegt dem prozeduralen Verständnis der Demokratie zugrunde. Große Mehrheiten und Einstimmigkeit scheinen für die Gemeinwohlorientierung einer Maßnahme zu sprechen. Gelegentlich wird die Opposition jedoch nur durch taktische Überlegungen zur Zustimmung bewogen, um in der Öffentlichkeit nicht dafür angeprangert zu werden, daß es in einer wichtigen Frage nicht zur Entscheidung kam. Die Größe der Mehrheit und die Gemeinwohlträchtigkeit von Gesetzen korrelieren auch sonst nicht.

\subsection{Die wissenschaftliche Vorbereitung zur Vermeidung von Schädigungen des Gemeinwohls und die Ergebnisse der ex-post-Evaluation}

Als Gegengewicht gegen die organisierten Interessen wird mit zunehmender Komplexität der Materien die wissenschaftliche Beratung im Gesetzgebungsprozeß wichtiger. Beiräte, Gutachten, Experten, die zu Hearings im Parlamentsausschuß eingeladen werden, sind vielfach funktionalisiert worden. Dabei kommt es zum Konflikt derer, die glauben, das Gemeinwohl sei wissenschaftlich exakt auszumachen und jenen, die sich antitechnokratisch gerade auf die Werte berufen, die nicht wissenschaftlich zu eruieren sind. Je tiefer das Gemeinwohl in einer Debatte auf Verträglichkeitstheorien herunteraggregiert wird, umso mehr gewinnt die wissenschaftsgläubige Seite die Oberhand. Das Gemeinwohl wurde vielfach auf die Formeln vom "Stand der Wissenschaft" und vom ,Stand der Technik" reduziert. Notfalls verpflichtet das Bundesverfassungsgericht den Gesetzgeber auf einen Stand der Wissenschaft. Gelegentlich sogar für die Zukunft, wie beim Volkszählungsurteil (BVerfGE 65, 55), als judiziert wurde, daß vorerst noch die umfassende Volkszählung nötig sei, in Zukunft aber die Stichprobenanalyse ausreichen könne.

Die Funktionalisierung des wissenschaftlichen Sachverstandes hat zugenommen. Aber sie hat schon immer existiert. Bei reformorientierten Routineentscheidungen wie beim Arbeiterrentenversicherung-Neuregelungsgesetz (ArVNG, 1957) operierten die

${ }^{45} \mathrm{Vgl}$. Beyme 1997, S. 43, $207 \mathrm{ff}$. 
Konfliktpartner ständig mit dem Vorwurf, die Gegenpartei gehe von einer veralteten wissenschaftlichen Theorie aus. ${ }^{46}$ Selbst klare Aussagen der Experten, wie beim Mitbestimmungsgesetz, ${ }^{47}$ wurden mit der Behauptung funktionalisiert, alle Experten hätten an dem Entwurf kein gutes Haar gelassen. Dabei waren die Stellungnahmen sehr viel differenzierter, und gleichwohl ließen sich in der Frage der Einschätzung des Entwurfs als verfassungsmäßig oder verfassungswidrig klare Relationen angeben, die in den Medien ausführlich besprochen wurden.

Bei Innovationsentscheidungen, die Neuland betraten, wie in der Debatte um die Gentechnik, war der Konsens über die Geltungstiefe des Wissens am geringsten. Selbst die Expertenkommissionen wurden schnell mit dem Vorwurf bedacht, es seien nicht genügend ökologische Experten hinzugezogen worden und man habe ein „Übergewicht der potentiellen Nutznießer" in der Kommission sichergestellt. ${ }^{48}$

Die schmale Wissensbasis des Parlaments wurde in Plenardebatten angeprangert: „Das Gentechnikgesetz, das heute zur Abstimmung vorliegt, legalisiert wieder einmal auf der Basis lückenhaften Wissens die großflächige industrielle Anwendung einer neuen Hochrisikotechnologie. ${ }^{649}$ Den Kritikern wurde von den Verteidigern des Gesetzes häufig der Sachverstand abgesprochen, vor allem den Grünen. Diese konterten: „Die Befürworter der Gentechnik scheinen demgegenüber sozusagen naturgemäß mit hinreichendem Sachverstand ausgestattet zu sein." ${ }^{50}$ Die Regierung berief sich auf Präzedenzfälle. Es sei erst in zwei Monaten die erste Freisetzung geplant. Die 167 Freisetzungen, die es bisher unter wissenschaftlicher Beobachtung anderwärts gegeben habe, seien ohne negative Folgen geblieben. ${ }^{51}$ Man verschanzte sich in dieser Debatte zunehmend hinter den Erfahrungen in Amerika und den Richtlinien der EU. Die Opposition rekurrierte nicht auf verharmlosende Präzedenzfälle, sondern rechnete die Technikfolgen in der Zukunft aus. Nach dem Vorschlag der Regierungskoalition wären 963 der Anlagen ohne Öffentlichkeitsbeteiligung und nur 23 mit Beteiligung der Öffentlichkeit genehmigt worden. ${ }^{52}$ Die Rechnung gipfelte in dem Vorwurf schlichter Verfassungswidrigkeit. Im Streit um die Wertakzeptanz wurden vielfach die Großen der Geschichte als Zeugen für und wider aufgerufen. Als der parlamentarische Staatssekretär im Justizministerium, Jahn, in der Debatte um das Embryonenschutzgesetz, ein Zitat aus Faust II über die Schaffung des Homunculus anführte („Was man an der Natur geheimnisvalles pries, das wagen wir verständig zu probieren, was sie sonst organisieren hieß, das lassen wir kristallisieren."), um das Parlament zu ermahnen „die richtigen Schranken zu setzen“, kam die Zwischenfrage von der Opposition (Westphal, SPD): „Ich wollte Sie nur fragen, ob Sie meinen, daß Goethe diesem Ge-

\footnotetext{
46 2.WP. 18.1.1957, 10424, Kalinke, DP; ebd. 10426, B. Preller, SPD.

47 7.WP. 18.3.1976, 16003 A.

48 11. WP. 29.3.1990, 15974 D, Frau Bulmahn, SPD.

49 Ebd., 15964 B, Frau Rust, Grüne.

50 Ebd., 15965 A.

51 Ebd., 15972 A.

s2 Ebd., 15975 C.
} 
setz zugestimmt hätte? Ich glaube, daß er es nicht getan hätte“ (Heiterkeit und Beifall bei der SPD). ${ }^{53}$

Alle Parteien haben sich zur wissenschaftlichen Grundlage der Politik bekannt. Aber die Wissenschaftsgläubigkeit war nicht bei allen Parteien gleich stark entwickelt. Sie galt als am stärksten bei der FDP und am schwächsten bei der CSU. ${ }^{54}$ Aber auch die CSU bekannte: „Die CSU ist eine politische Aktionsgemeinschaft, die wissenschaftliche Erkenntnisse von den Veränderungen unserer Umwelt politisch verantwortlich verwirklicht." ${ }^{55}$ Das entscheidende Moment dieses Satzes ist die Einschränkung ,politisch verantwortlich". Die Politiker erfahren täglich, daß Wissenschaftler nicht einer Meinung sind, und plädieren für Vorsicht gegenüber einer wissenschaftlichen Festlegung des Gemeinwohls. ${ }^{56}$ In der Rechtspolitik wurde vielfach das „Rechtsempfinden des Volkes" als Grenze angesehen. Das Bekenntnis zur Wertentscheidung im Gegensatz zu wissenschaftlicher Fortschrittsgläubigkeit gipfelte in dem Satz von Max Güde: ${ }^{57}$ „Das Recht hat nicht die Aufgabe, Vorreiter und Wegbereiter der sexuellen Revolution zu sein."

Wissenschaftliche Expertise wurde vielfach unter Anrufung eines werthaften Gemeinwohls, das auf Akzeptanz im Volk ruhte, verworfen mit Gründen wie mangelnde Akzeptanz im Volk, ethische Bedenken, die durch Ethikkommissionen nicht ausgeräumt werden konnten, Nichtimplementierbarkeit eines Vorschlags, das Fehlen finanzieller Ressourcen und Unwägbarkeiten von Risiken aufgrund unvorhergesehener Nebenfolgen.

Gemeinwohl, auch reduziert als Natur-, Umwelt- oder Sozialverträglichkeit, verlangt in diesen Fällen ein staatliches Nichthandeln. Erst, wenn das Nichthandeln seinerseits zu Krisen oder Unglücken führte, wird in einer Gegenwelle der wissenschaftliche Sachverstand zum Hüter des Gemeinwohls erklärt und forciertes staatliches Handeln angemahnt.

Die Tatsache, daß wichtige Gesetze ex post facto evaluiert werden müssen, zeigt, daß die ex ante-Vorbereitung von Maßnahmen durch wissenschaftliche Expertise nicht ausreicht. Die Einrichtung der Evaluation durch Berichtspflichten, Kommissionen und Rechnungshöfe demonstriert, daß Demokratien fallibilistisch vorgehen. Zudem haben Theorien der Risikogesellschaft die Gemeinwohlträchtigkeit der meisten weitreichenden Entscheidungen in Frage gestellt.

Es wird geltend gemacht, daß nur wahrgenommene Risiken reguliert werden. Hypothetische Risiken ließen sich politisch nicht durchsetzen und würden rechtlicher Nachprüfung bei Klagen nicht standhalten.

Risiken, die bekannt sind, haben gleichwohl häufig unklare Folgen, da die lineare Kausalität nicht mehr anwendbar ist. Unklare Vorausschau von Risiken führt zu unbestimmten Rechtsbegriffen, die jeder Gesetzgebung abträglich sein müssen.

53 11. WP. 24.10.1990, 18218 A.

54 Vgl. Müller 1977, S. 295.

55 CSU-Grundsatzprogramm 1968.

56 5. WP, 12835 C, Müller-Emmert, SPD.

57 5. WP 9.5.1969, 12832 D. 
Das Recht kann als wirksam angesehen werden, aber es wird befürchtet, daß jede Normierung zu spät kommt. Gesetzgebung droht mehr und mehr, nur die Milderung längst eingetretener Schäden zu regulieren.

Der Gesetzgeber ist angesichts dieser Dilemmata überfordert, verzichtet auf rechtliche Gestaltung einer Materie und überläßt die Technikfolgen der Exekutive.

Risiko und Gefährdung - von Ulrich Beck unzureichend differenziert - werden im Sprachgebrauch der Versicherungswirtschaft ebenfalls nicht streng genug auseinander gehalten. Risiko hat ein ähnliches Ausmaß an Schaden, birgt aber eine geringere Wahrscheinlichkeit des Eintritts eines schädigenden Ereignisses als die normale Gefährdung. Die Betroffenen aber können das Ausmaß der Gefährdung oder des Risikos kaum je realistisch einschätzen. Öko-Populismus plus Medienverstärkung können jederzeit kollektive Hysterien bei geringer Gefährdung erzeugen. Der Gesetzgeber ist daher gut beraten, von einem relativ laxen Risikobegriff auszugehen, der Restrisiken einkalkuliert. In diesem Sinne judizierte auch das Bundesverfassungsgericht.

Das Gemeinwohl wird vielfach widersprüchlich durch Widersprüche zwischen Grundrechten. Der klassische Konflikt zweier Grundrechte ist auch in den Risikotechnologien nicht aufgehoben und kann nicht durch Maximierung eines Ziels gelöst werden. Das Grundgesetz stellt keine eindeutig richtigen Antworten für die Lösung des Konflikts im Einzelfall bereit. ${ }^{58}$

Leichter geworden scheint die Evaluierung, weil neuere Gesetze immer feinere Arsenale von Anzeigepflichten, Untersagungsermächtigungen und Verboten mit Erlaubnisvorbehalten einsetzen. Sie alle umgreift, wenn auch vielfach in vager und damit schwer evaluierbarer Form bei den Folgen von Gesetzen, die einleitende Skizzierung des Zwekkes der Gesetze, einschließlich der Beschreibung des Ziels, eines gewünschten Zustandes in der Zukunft. Dies begann etwa beim Chemikaliengesetz (1980) und bei späteren Umweltschutzgesetzen. Damit drängt die Gesetzgebung weiter in Richtung von Programmgesetzen mit finaler Zwecksteuerung, obwohl die Autopoietiker gerade davor dringend warnen. Je umfassender die Gesetzesvorhaben, wie beim Raumordnungsgesetz (1965) und bei den großen Kodifikationen, etwa dem Sozialgesetzbuch (allgemeiner Teil 1975), desto stärker kam es gelegentlich zu Formulierungen, die auch Juristen eher ,phrasenhaft" dünkten.

Die Instrumente, die dem Schutzzweck dienen sollen, sind unterschiedlicher Natur: von dem Verbot mit Erlaubnisvorbehalt (BImSchG) bis zur Konstituierung von Eigentum der Gemeinschaft an besonderen spaltbaren Stoffen im Atomgesetz nach dem Euratom-Vertrag (1957 Art. 87). Was bisher unter Besatzungsrechtsvorbehalt gestanden hatte, wurde nun vom deutschen Gesetzgeber erstmals erschlossen. Förderzwecke traten somit in den Vordergrund. Förder- und Schutzzweck gerieten vielfach in Konflikt. Verwaltungsgerichtsurteile $^{59}$ gaben dem Schutzzweck zum Teil Vorrang. Das Verfassungsgericht hat im Kalkar-Urteil ${ }^{60}$ den Vorrang des Schutzzwecks relativiert auf den Bereich empirisch gesicherter Schadenswahrscheinlichkeiten. Nach bestmöglicher Gefahrenabwehr und Risikovorsorge ist ein Restrisiko von allen Bürgern gemeinsam zu

\footnotetext{
$58 \mathrm{Vgl}$. Vitzthum/Geddert-Steinacher 1992, S. 16.

59 BVerwG, DVBI 1972, S. 678, Urteil zu Würgassen.

${ }^{60}$ BVerfGE S. $49,89,141 \mathrm{ff}$.
} 
tragen. Das Gemeinwohl ist auch nach vielen Gerichtsurteilen für die Zukunft nicht mit absoluter Verläßlichkeit zu erkennen.

Der Staat begibt sich vielfach in die prekäre Zone der Selbstüberforderung, weil er die punktuelle Sicherheitskontrolle zu einer umfassenden Risikovorsorge erweitert. ${ }^{61}$ Wertkonflikte sind in Gesetzen angelegt. Auch bei der Gentechnik wurde eine Grundrechtskonkurrenz von Schutz- und Förderungsgedanken angelegt, obwohl das Gentechnikgesetz eher dem klassischen Typ der Gefahrenabwehr als dem der „,betätigungseröffnenden Wirtschaftsgestaltung" zugerechnet worden ist.

Viele der programmatischen Aussagen der Zweck- und Zieldefinitionen sind kaum vollzugsreif. Seit dem BImSchG (1974) wird praktiziert, dies durch eine Flut von Verordnungen und Satzungen zu erreichen, welche die Frage nach dem Parlamentsvorbehalt für so weit reichende Regelungen aufwerfen. In anderen Fällen, wie beim Embryonenschutzgesetz (1990), drohte die Gefahr, daß ein durch Interesseneinflüsse geschwächter Schutzgedanke in symbolischer Politik endet, weil regelrecht verboten nur das erschien, was in absehbarer Zeit ohnehin niemand machen kann. ${ }^{62} \mathrm{Je}$ hochtrabender die Zwecke eines Gesetzes formuliert wurden, desto stärker bleibt ihre Wirkung hinter den Erwartungen des Gesetzgebers zurück. Gentechnik und Embryonengesetz (1990) waren Teillösungen eines großen Regelungskomplexes. Sowohl die Opposition als auch einige Länder, wie Niedersachsen, Bayern oder Hessen, in denen unterschiedliche Koalitionen regierten, forderten ein umfassendes Gesetz zur Fortpflanzungsmedizin. Aber es wäre ohne Grundgesetzänderung (Art. 74) kaum zu realisieren gewesen. Die Aufsplitterung der Materie machen sie durchsetzungsfähiger, aber auch schwerer in ihrer Wirksamkeit zu evaluieren, weil zahlreiche Regelungslücken zurückblieben.

Demokratische Entscheidungen in fragmentierten Systemen des semisouveränen Staates, in dem organisierte Interessen, Parteien, Gebietseinheiten, Bürokratien und parastaatliche Einrichtungen einander kontrollieren und lähmen, spaltet die Materien auf und splittert das Gemeinwohl in lauter kleine Gemeinwöhlchen auf, die als Verträglichkeitsannahmen und Risikovermeidungsmaximen bescheiden geworden sind.

Viele Kriterien werden evaluiert: von der Bezahlbarkeit bis zur Durchführbarkeit einer Maßnahme. Kein Kriterium aber ist so stark, wie die Akzeptanz. Akzeptanz ist eine notwendige, aber keine hinreichende Bedingung für sozialverträgliche Technikgestaltung. ${ }^{63}$ Techniken, die aufgrund der kulturellen Traditionen eines Landes wie den USA akzeptiert werden, lösen in anderen Systemen, wie Deutschland, kollektive Hysterie aus, die erst mit Hinweis auf die Bewährung in Amerika von den Technokraten langsam abgebaut zu werden pflegt. Wo abgehobene Parteipolitiker in die Kritik geraten, ist die Versuchung groß, Responsivität in der Stimmungsdemokratie auszubauen. Akzeptanz wird dann gern mit dem Gemeinwohl gleichgesetzt. Je komplexer die Materien, umso weniger kann der Bürger sie jedoch einschätzen.

Akzeptiert wird vielfach, was nicht durch eine Medien- oder Interessengruppenkampagne mit einem Veto bedacht wird. Spezialistengesetze, wie das Immissionsschutzge-

61 Vitzthum 1990, S. 24.

${ }^{62}$ Vgl. Gill 1991, S. 110.

${ }^{63} \mathrm{Vgl}$. Martinsen 1997, S. 220. 
setz (1974) oder das Chemikaliengesetz (1980) waren vielfach selbst den Experten unter den hauptbetroffenen Industrien in ihren Folgen nicht deutlich. Die Gegenmobilisierung setzte erst bei den Ausführungsbestimmungen ein.

Dennoch zeigt sich immer wieder, daß der Konsensbedarf einer Gesellschaft überschätzt wird. Seit Easton weiß die Legitimationsforschung, daß eher generalisierte Erwartungen und diffuse Unterstützungsbereitschaft ein System am Leben halten als explizite Zustimmung. Daher können die politischen Eliten es sich in wichtigen Fragen, wie bei der Strafgesetzgebung, immer wieder leisten, die Lufthoheit über den Stammtischen zu verletzen und gegen die Volksmehrheitsmeinung zu entscheiden. Gemeinwohl entpuppt sich somit nicht als ein Punkt auf einer Maßnahmelinie, sondern als eine Vermutung des Grundkonsenses, der nur durch langanhaltende Strapazierung der Akzeptanz verletzt werden kann. Aber auch in diesem Fall ist die Folge nicht Rebellion, sondern ein Wandel in der Zusammensetzung der Regierung.

\section{Fazit}

Es gibt in politischen Prozessen unterschiedliche Formen der Gemeinwohlrhetorik, die das Wortfeld semantisch gruppengerecht absteckt. Aber es gibt auch Gemeinwohlorientierung. Der eigennützigste Verband muß sich die eigene Gemeinwohlrhetorik zurechnen lassen. Je kooperativer das System der Verbände und Parteien ist, umso mehr wird das Gemeinwohl auch benutzt, um unvermeidliche Kompromisse der eigenen Mitgliedschaft schmackhaft zu machen und die Konzessionen nicht als Niederlage der Gruppenführung erscheinen zu lassen. Daher akzeptieren Parteien und Verbände in der Demokratie Entscheidungen gegen sich und mildern sie rhetorisch $a b$ - in einer Art „Gemeinwohlselbsttröstung“.

\section{Literaturverzeichnis:}

Abromeit, H. (1993), Interessenvermittlung zwischen Konkurrenz und Konkordanz, Opladen. Alemann, U. v./Heinz, R. G. (Hg., 1979), Verbände und Staat, Opladen.

Almond, G. A./Verba, S. (Hg., 1965), The Civic Culture. Political Attitudes and Democracy in Five Nations, Boston.

Arnim, H.-H. v. (1977), Gemeinwohl und Gruppeninteressen, Frankfurt/M.

Bentham, J. (1948), The Principle of Morals and Legislation [1780], New York.

Bentley, A. F. (1949), The Process of Government. A study of social pressures [1908], Evanston/IIl.

Beyme, K. v. (1997), Der Gesetzgeber. Der Bundestag als Entscheidungszentrum, Opladen.

Dohnanyi, K. v. (1999), In weiter Distanz. Klaus von Dohnanyi über Pragmatiker und Sozialdogmatiker in der SPD, in: Der Spiegel 49/1999, S. 40-41.

Eppler, E. (1984), Grundwerte für ein neues Godesberger Programm, Reinbek.

Eschenburg, T. (1955), Herrschaft der Verbände?, Stuttgart

Etzioni, A. (1995), Die Entdeckung des Gemeinsinns, Stuttgart.

Falaquera, Shem Tov Ibn (1976), Book of the Seeker [1263], New York.

Fraenkel, E. (1964), Deutschland und die westlichen Demokratien, Stuttgart.

Geißler, H. (Hg., 1979), Grundwerte in der Politik, Berlin. 
Gill, B. (1991), Gentechnik ohne Politik, Frankfurt/M.

Göhner, R. (1981), Demokratie in Verbänden, München

Groser, M. (1992), Gemeinwohl und Ärzteinteressen - die Politik des Hartmannbundes, Gütersloh.

Häberle, P. (1970), Öffentliches Interesse als juristisches Problem. Eine Analyse von Gesetzgebung und Rechtsprechung, Bad Homburg v.d.H.

Heinze, R. G. (1992), Verbandspolitik zwischen Partikularinteressen und Gemeinwohl - der Deutsche Bauernverband, Gütersloh.

Herzinger, R. (1997), Die Tyrannei des Gemeinsinns, Berlin.

Hirschman, A. (1984), Engagement und Enttäuschung. Über das Schwanken der Bürger zwischen Privatwohl und Gemeinwohl, Frankfurt/M.

Höffe, O. (1995), Gerechtigkeit, in: Politische Theorien. Lexikon der Politik, Bd. 1, hg. v. D. Nohlen u. R.-O. Schultze, München, S. 144-152.

Hondrich, K.-O. (1963), Die Ideologien von Interessenverbänden, Berlin.

Mandeville, B. (1968), Die Bienenfabel [1723], Frankfurt/M.

Martinsen, R. (Hg., 1997), Politik und Biotechnologie, Baden-Baden.

Mayntz, R. (Hg., 1992), Verbände zwischen Mitgliederinteressen und Gemeinwohl. Gütersloh.

Meyer, T. (Hg., 1981), Grundwerte und Geseilschaftsreform, FrankfurtM.

Miller, D. u. a. (Hg., 1993), The Blackwell Encyclopedia of Political Thought, Oxford.

Müller, T. (1977), Die Haltung der Parteien in der Bundesrepublik Deutschland zu den Problemen von Strafe und Strafvollzug, Frankfurt/M. etc.

Münkler, H./Llanque, M. (Hg., 1999), Konzeptionen der Gerechtigkeit, Baden-Baden.

Olson, M. (1968), Die Logik des kollektiven Handelns, Tübingen.

Rawls, J. (1979), Eine Theorie der Gerechtigkeit, Frankfurt/M

Reese-Schäfer, W. (1997), Grenzgötter der Moral. Der neuere europäisch-amerikanische Diskurs zur politischen Ethik, Frankfurt/M.

Riker, W. H./Ordeshook, P. C. (1973), An Introduction to Positive Political Theory, Englewood Cliffs.

Ronge, V. (1992), Vom Verbändegesetz zur Sozialverträglichkeit - Die öffentliche und verbandliche Diskussion über den Gemeinwohlbezug von Verbänden in den 80er Jahren, in: Verbände zwischen Mitgliederinteressen und Gemeinwohl, hg. v. R. Mayntz, Gütersloh, S. 36-79.

Sarcinelli, U. (1979), Das Staatsverständnis der SPD, Meisenheim.

Schubert, G. (1960), The Public Interest, Glencoe/IIl.

Schütt-Wetschky, E. (1997), Interessenverbände und Staat, Darmstadt.

Schwan, A. (1978), Grundwerte der Demokratie, München.

Schwan, A. u. a. $\left(1986^{7}\right)$, Gemeinwohl, in: Staatslexikon, Bd. 2, Freiburg, S. 857-863.

Teubner, G. (1978), Organisationsdemokratie und Verbandsverfassung, Tübingen.

Ulrich, B. (1999), Die Gerechtigkeitsfalle, in: Der Tagesspiegel, 25.09.1999, S. 33.

Vitzthum, W. Graf (1990), Der Zweck im Gentechnikrecht: zur Schutz- und Förderfunktion von Umweltund Technikgesetzen, Berlin.

Vitzthum, W. Graf/Geddert-Steinacher, T. (1992), Standortgefährdung. Zur Gentechnikregelung in Deutschland, Berlin.

Vobruba, G. (1992), Wirtschaftsverbände und Gemeinwohl, in: Verbände zwischen Mitgliederinteressen und Gemeinwohl, hg. v. R. Mayntz, Gütersloh, S. 80-121.

Weldon, T. D. (1953, 1960), The Vocabulary of Politics, Harmondsworth 